\title{
Cuidados de enfermagem relacionados à cardiotoxicidade envolvendo drogas como a antraciclinas e anticorpo monoclonais no tratamento oncológico
}

\author{
Nursing care related to cardiotoxicity involving drugs such as anthracyclines and monoclonal \\ antibodies in cancer treatment
}

\author{
Lorena Esmeralda Nascimento Celeste ${ }^{1 *} \bullet$, Maiara Rodrigues Maia $^{2} \bullet$
}

\begin{abstract}
${ }^{1}$ Enfermeira Preceptora na Universidade Salvador, Especialista em Atenção Básica e Docência do Ensino Superior, Salvador, Bahia, Brasil. ${ }^{2}$ Residente no programa de residência multiprofissional do hospital de beneficências de senhoras Sírio Libanês no programa de cuidado do paciente oncológico, São Paulo, São Paulo, Brasil.*Autor para correspondência. E-mail: lory.celeste@yahoo.com.br
\end{abstract}

\begin{abstract}
Resumo: Introdução: As doenças cardiovasculares nos pacientes com câncer são eventos cada vez mais frequentes, em decorrência de avanços na terapêutica oncológica que resultaram tanto na melhora da qualidade de vida como no aumento da sobrevida dos pacientes. Nas últimas décadas, os progressos no tratamento oncológico resultaram também na maior exposição dos pacientes a fatores de risco cardiovasculares e à quimioterapia com potencial de cardiotoxicidade. Revisão: Trata-se da realização de um trabalho de estudo de revisão bibliográfica do tipo integrativo, destinada a avaliar os cuidados de enfermagem no uso de antineoplásicos como efeitos adversos principal a cardiotoxicidade e relatar a gravidade do uso das antraciclinas e do anticorpo monoclonal. Discussão: As antracilinas são antibióticos antitumorais, amplamente utilizados em diversos tipos de câncer e os anticorpos monoclonais é um tipo de terapia imunológica, podendo ser combinada com os tratamentos convencionais, pois também é um antitumoral. Ambos constituem uma reconhecida causa de cardiotoxicidade. Considerações finais: É de fundamental relevância que a enfermagem atue antes, durante e após a administração da quimioterapia, observando os sinais e sintomas e orientando ambos, tanto o paciente quanto o familiar sobre o antineoplásico utilizado, sua ação no organismo, seus efeitos colaterais e os cuidados em geral.
\end{abstract}

Palavras-chave: cardiotoxicidade, antraciclinas, anticorpo monoclonal, cuidados de enfermagem.

\begin{abstract}
Introduction: Cardiovascular diseases in cancer patients are increasingly frequent events, due to advances in cancer therapy that have resulted both in improving quality of life and increasing patient survival. In recent decades, progress in cancer treatment has also resulted in increased exposure of patients to cardiovascular risk factors and chemotherapy with potential for cardiotoxicity. Review: This is a work of bibliographic review of the integrative type, aimed at evaluating nursing care in the use of antineoplastic as main adverse effects to cardiotoxicity and reporting the severity of the use of anthracycline and monoclonal antibody. Discussion: Anthracyclines are antitumor antibiotics, widely used in various types of cancer and monoclonal antibodies is a type of immunological therapy and can be combined with conventional treatments, as it is also an antitumor. Both are a recognized cause of cardiotoxicity. Final considerations: It is of fundamental importance that nursing act before, during and after chemotherapy administration, observing the signs and symptoms and guiding both the patient and the family member about the antineoplastic used, its action on the body, its side effects and care in general.
\end{abstract}

Keywords:cardiotoxicity, anthracyclines, monoclonal antibody, nursing care.

\section{Introdução}

O envelhecimento natural do ser humano traz mudanças nas células, que aumentam a sua suscetibilidade à transformação maligna. Isso, somado ao fato de as células das pessoas idosas terem sido expostas por mais tempo aos diferentes fatores de risco para câncer, explica, em parte, o porquê de o câncer ser mais freqüente nessa fase da vida. No Brasil, a mortalidade por neoplasias vem crescendo consideravelmente ao longo das últimas décadas, ao mesmo tempo em que diminuíram as mortes por doenças infecto parasitárias (INCA, 2020).

Segundo os dados da Organização Mundial de Saúde, as patologias do foro oncológico constituem, na atualidade, a segunda maior causa de morte no mundo, nas duas primeiras décadas do século passado, 
enquanto as endemias ocupavam a atenção das políticas de saúde no Brasil, o câncer começava a despontar nos países desenvolvidos entre as doenças de maior taxa de mortalidade.

Doenças e agravos não transmissíveis são as principais causas de adoecimento e mortes na população mundial. Estima-se que em 2008, ocorreram 36 milhões de mortes decorrentes dessas doenças, com destaque para as doenças cardiovasculares e câncer, e tendo maior impacto nos países pouco desenvolvidos. As transições demográficas e epidemiológicas das últimas décadas apontam o câncer com maior carga nas próximas décadas de acordo com Borges et al. (2018).

O tratamento atual do câncer utiliza uma combinação de quimioterapia, radioterapia e cirurgia, os quais podem prolongar a vida e trazer a cura da doença. Alguns desses tratamentos podem precipitar o aparecimento ou o agravamento de doença cardiovascular (Filho \& Gonçalves, 2011).

As doenças cardiovasculares nos pacientes com câncer são eventos cada vez mais frequentes, em decorrência de avanços na terapêutica oncológica que resultaram tanto na melhora da qualidade de vida como no aumento da sobrevida dos pacientes. Nas últimas décadas, os progressos no tratamento oncológico resultaram também na maior exposição dos pacientes a fatores de risco cardiovasculares e à quimioterapia com potencial de cardiotoxicidade de acordo com Araujo et al. (2018).

Segundo Cruz et al. (2016) apud Zata et al. (2014) Asantraciclinas como a doxorrubicina, daunorrubicina, epirrubicina, mitoxantrona, adriamicina-ADR e idarrubicina estão entre os agentes mais ativos dos quimioterápicos mais usados na terapêutica farmacológica do câncer e em esquema de combinação da melhora a sobrevida livre de doença e global nos pacientes, e constituem uma reconhecida causa de cardiotoxicidade.

Regimes de quimioterapia sem antraciclinas apresentam taxas menores de disfunção cardíaca quando comparados com regimes de uso combinado de trastuzumab e antraciclinas. Um dos principais fatores de risco cardiotóxicos associados ao uso antineoplásico de trastuzumab é a utilização de elevadas doses cumulativas de antraciclinas (> $300 \mathrm{mg} / \mathrm{m} 2$ ) (Adão et al., 2013).

A escolha pelo tema veio de tentar alcançar o objetivo geral de Realizar uma revisão de literatura sobre o cuidado de enfermagem na introdução de antineoplásicos a base de antraciclinas e alguns anticorpos monoclonais cardiotóxicos, no qual tem o intuito de contribuir com informações e dados relevantes nos índices de atendimento ao paciente oncologico no uso de drogas com potencial cardiotoxicidade e a conscientização da importância do suporte multiprofissional no manejo desse paciente com potencial risco de danos agudos e crônicos.

Devido o tão quanto a temática é importante buscamos elencar dois objetivos específicos, os de Descrever as drogas de maior impacto cardiológico no uso em pacientes em tratamento oncológico, e o de Analisar os cuidados de enfermagem, frente à administração de drogas cardiotóxicas como fatores de toxicidade aguda e crônica na oncologia.

Diante da relevância que o tema nos remete é importante deixar claras as drogas envolvidas nessa toxicidade, o risco que pode acarretar ao longo da vida além do tratamento propriamente dito, então de acordo com as anuências que o assunto pode transmitir, esse artigo tem a intenção de realizar o embasamento teórico, necessário para o manejo de pacientes que estejam em uso desses devidos antineoplásicos.

\section{Revisão}

Trata-se da realização de um trabalho de estudo de revisão bibliográfica do tipo integrativo, destinada a avaliar os cuidados de enfermagem no uso de antineoplásicos como efeitos adversos principal a cardiotoxicidade e relatar a gravidade do uso das antraciclinas e do anticorpo monoclonal.

A revisão de literatura (ou revisão integrativa) é sempre recomendada para o levantamento da produção científica disponível e para a reconstrução de redes de pensamentos e conceitos, que articulam saberes de diversas fontes na tentativa de trilhar caminhos na direção daquilo que se deseja conhecer (Gomes \& Caminha, 2014).

Será uma pesquisa descritiva, do ponto de vista dos objetivos (segundo Gil, 1994) a pesquisa do ponto dos procedimentos técnicos é bibliográfica segundo Selltiz et al. (1967) ,apud (Gil, 2002) elaborada a partir de material já publicado, como livros, artigos, periódicos, Internet.

Para o levantamento dos artigos, foi realizada uma revisão integrativa por meio de pesquisa utilizada nas bases de dados eletrônica SCIELO, LILACS e BDENF. 
Foram utilizados nas bases de dados os descritores (e/ou unitermos) em português "cardiotoxicidade" e "antraciclinas" e "anticorpo monoclonal" e "cuidados de enfermagem" realizando-se a busca destes termos no título, resumo ou corpo do artigo.

Após realizar este levantamento, optamos identificar uma primeira seleção a partir dos títulos, depois a leitura dos resumos e, após, procederemos à seleção e leitura na íntegra dos artigos a serem analisados. No qual iniciamos a produção da revisão nos meses de maio de 2020 até Setembro de 2020, alguns critérios de inclusão foram títulos, resumos e estudos observacionais, e critérios de exclusão os estudos repetidos entre as bases, os indisponíveis na íntegra e os que não abordem o tema central deste estudo, ou estudos de revisão de literatura, sem resumos, trabalhos de conclusão de curso e relatos de caso foram excluídos.

\section{Discussão}

\section{Drogas de maior impacto cardiotóxico em pacientes em tratamento oncológico}

As doenças cardiovasculares nos pacientes com câncer são eventos cada vez mais freqüentes em decorrência de avanços na terapêutica oncológica, que resultaram tanto na melhora da qualidade de vida como no aumento da sobrevida dos pacientes. Atualmente, observa-se uma mudança no paradigma em relação ao prognóstico do paciente oncológico, que passa a ser visto como um portador de uma doença crônica que ao longo de sua evolução pode apresentar descompensações agudas, como as manifestações cardiovasculares (Filho et al., 2017).

Nas últimas décadas os tratamentos antineoplásicos têm evoluído drasticamente e melhoraram significativamente o prognóstico dos pacientes oncológicos. Porém, o uso contínuo dos agentes quimioterápicos na prática clínica pode gerar controvérsia, devido aos seus potenciais efeitos adversos ao nível cardiovascular nos pacientes tratados que sobrevivem à doença oncológica (Adao et al., 2012).

As antracilinas são antibióticos antitumorais, amplamente utilizados em diversos tipos de câncer e os anticorpos monoclonais é um tipo de terapia imunológica, podendo ser combinada com os tratamentos convencionais, pois também é um antitumoral. Ambos constituem uma reconhecida causa de cardiotoxicidade (Tabela 1).

Tabela 1.Cardiotoxidade das principais classes de fármacos antineoplásicos.

\begin{tabular}{|c|c|c|c|}
\hline Classificação Farmacêutica & Classe & Drogas & Toxicidade Cardiovascular \\
\hline Antibiótico Antitumoral & Antraciclinas & $\begin{array}{l}\text { Doxorrubicina } \\
\text { Epirrubicina } \\
\text { Daunorrubicina } \\
\text { Idarrubicina } \\
\text { Mitoxantrone }\end{array}$ & $\begin{array}{l}\text { Aguda: IC, arritmias, alterações no } \\
\text { intervalo Q-T, alteração na } \\
\text { repolarização ventricular. } \\
\text { Crônica (dose-dependente): } \\
\text { Disfunção ventricular esquerda (não } \\
\text { reversível). }\end{array}$ \\
\hline Anticorpo Monoclonal & $\begin{array}{l}\text { Anticorpos inibidores da } \\
\text { tirosina-cínase }\end{array}$ & $\begin{array}{l}\text { Trastuzumab } \\
\text { Bevacizumab } \\
\text { Rituximab } \\
\text { Alemtuzumab }\end{array}$ & $\begin{array}{l}\text { Disfunção ventricular esquerda } \\
\text { (reversível) } \\
\text { Hipertensão arterial sistêmica, } \\
\text { tromboembolismo venoso, disfunção } \\
\text { ventricular esquerda } \\
\text { Hipotensão ortostática, isquemia } \\
\text { miocárdica, disfunção ventricular } \\
\text { esquerda. }\end{array}$ \\
\hline
\end{tabular}

Fonte: Dados da pesquisa. Legenda: IC - Insuficiência Cardíaca.

Antibióticos antitumorais é um grupo de substâncias com estrutura química variada que, embora interajam com o DNA e inibam a síntese deste ácido ou de proteínas, não atuam especificamente sobre determinada fase do ciclo celular. Apesar de apresentarem tal variação, possuem em comum anéis insaturados que permitem a incorporação de excesso de elétrons e a conseqüente produção de radicais livres reativos (Bonassa, 2012).

Segundo Filho et al. (2017) dentre os efeitos adversos dos quimioterápicos no sistema cardiovascular destaca-se, pela sua maior freqüência e gravidade, a agressão miocárdica com disfunção ventricular sistólica e insuficiência cardíaca. O aparecimento dessa complicação pode determinar interrupção do tratamento quimioterápico e comprometer a cura ou o adequado controle do câncer. É válido ressaltar que a insuficiência cardíaca tem pior prognóstico que muitas neoplasias e pode comprometer seriamente a evolução do paciente em tratamento.

A abordagem da doença cardiovascular em pacientes com câncer é muitas vezes diferente da população em geral, não apenas por causa de mecanismos subjacentes distintos e características clínicas de suas 
doenças cardíacas, mas também por causa da potencial necessidade contínua de tratamento adicional do câncer, bem como a duração alterada da sobrevida antecipada. Em um esforço para maximizar a qualidade de vida e a sobrevivência, cardiologistas e oncologistas devem colaborar com o objetivo de equilibrar os riscos da cardiotoxicidade com os benefícios da terapia oncológica de acordo com Araujo et al. (2018).

A toxicidade cardiovascular dos quimioterápicos está associada ao período de exposição e à concentração plasmática da droga. Fatores de risco como, extremos de idade, radioterapia mediastinal, HAS, DM, disfunção ventricular prévia, uso de quimioterápicos associados e suscetibilidade genética, auxiliam para o aumento da cardiotoxicidade.

A cardiotoxicidade aguda induzida pelas antraciclinas é rara, transitória e independente da dose utilizada. Caracterizam-se por alterações súbitas da repolarização ventricular, alterações eletrocardiográficas no intervalo Q-T, arritmias ventriculares e supraventriculares e síndromes coronárias agudas. Estas alterações são geralmente observadas desde o início do tratamento até 14 dias após o término do mesmo (Adao et al., 2012).

A forma crônica depende da dose utilizada, podendo ocorrer nos primeiros anos após finalização do tratamento ou pode ocorrer tardiamente após um ano do tratamento. Os sintomas da cardiotoxicidade crônica é a disfunção ventricular esquerda, podendo ocasionar uma cardiomiopatia grave, podendo levar a morte.

Segundo Bonassa (2012) o anticorpo monoclonal Trastuzumabe (Herceptin), indicado para o tratamento de pacientes com câncer de mama metastático que apresentam tumores com superexpressão do HER2, pode levar a falência cardíaca moderada a grave, principalmente em mulheres que já receberam antracíclicos e ou ciclofosfamida.

Os anticorpos monoclonais, por sua vez, produzem alterações miocárdicas menos graves, sendo em grande parte reversíveis. A associação de antraciclinas e anticorpos monoclonais podem provocar cardiotoxidade com uma incidência maior de 40\%, especialmente em pacientes idosos (Fassio \& Castillo, 2016).

$\mathrm{O}$ anticorpo monoclonal são proteínas usadas pelo sistema imunológico para identificar e neutralizar corpos estranhos, como bactérias, vírus ou células tumorais. Um anticorpo reconhece um alvo especifico o antígeno, presente nas células estranhas ao organismo. As células neoplásicas expressam uma variedade de antígenos que representam alvos atraentes para a terapia baseada em anticorpos monoclonais (Bonassa, 2012).

Segundo Zata et al. (2014) É de fundamental importância no primeiro dia da infusão do quimioterápico com antracíclico, a enfermagem realizar o pré-condicionamento remoto isquêmico no paciente sentado, utilizando um manguito com um manômetro devidamente calibrado e certificado.

O tratamento da insuficiência cardíaca no paciente portador de neoplasia segue os mesmos princípios observados em um paciente não portador de câncer. Existe uma tendência de uma abordagem menos agressiva, em função do paciente já apresentar uma doença grave. Os betabloqueadores e os inibidores da enzima de conversão do angiotensinogênio continuam a ser basilares no tratamento medicamentoso (Filho \& Gonçalves, 2011).

\section{Cuidados de enfermagem frente à administração de drogas cardiotóxicas}

A Enfermagem tem papel fundamental, pois atua diretamente na assistência ao paciente oncológico com o objetivo de propiciar-lhe conforto, segurança e qualidade de vida, além de implementar ações de controle de riscos e problemas cardiotóxicos potenciais. Entretanto, a assistência a esses pacientes precisa ser pautada em evidências científicas, pois a cardiotoxicidade sem o efetivo, acompanhamento e manejo pode comprometer a eficácia do tratamento oncológico e/ou determinar a sua interrupção, comprometendo a cura do câncer ou o seu controle (Jesus, 2019).

Os pacientes em tratamento quimioterápico devem ser avaliados do ponto de vista cardíaco para estabelecer um padrão basal cardiovascular, que permita a comparação durante o regime terapêutico. Esta avaliação deve incluir: elaboração de história clínica e exame físico; realização de um eletrocardiograma (avaliação do ritmo cardíaco e detecção de sinais de isquemia) e exame de imagem cardíaca. Durante os regimes terapêuticos é importante monitorizar os sinais e sintomas de cardiotoxicidade, as alterações clínicas, sinais vitais, medidas antropométricas e estar atento as intercorrências e efeitos colaterais durante a administração das drogas, além de realizar as anotações de enfermagem (Cruz et al., 2016).

Portanto a administração de quimioterápicos está corelacionada ao potencial risco cardiotóxico e é de suma relevância que a enfermagem, ao realizar o cuidado, examine o paciente antes, durante e após administração dos antineoplásicos, com a finalidade de avaliar qualquer alteração clínica com indícios de cardiotoxicidade, ou até mesmo seus sintomas pré - clínicos.

A equipe de enfermagem tem que saber encorajar estes pacientes a manterem seu bem estar e fazer com que a esperança nunca desapareça, além de coordenar as ações de prevenção, diagnóstico e tratamento dos 
pacientes. É preciso oferecer alternativas de conforto, educação e assistência de qualidade, mantida na cumplicidade, respeito e dedicação (Zata et al., 2014).

\section{Considerações finais}

O número de casos de câncer no Brasil vem aumentando significativamente e devido aos inúmeros meios de tratamentos antineoplásicos, como a quimioterapia, vem aumentando a sobrevida dos pacientes.

Entretanto o uso dos quimioterápicos pode desenvolver certas toxicidades, com isso, o conhecimento prévio de certas drogas e da sua farmacocinética e farmacodinâmica, influenciam no desfecho dos eventos adversos, abordando os prováveis sinais e sintomas de cardiotoxidade, além dos fatores de risco.

É essencial a verificação do correto registro das informações pela enfermagem, tornam-se imprescindíveis. Também, notou-se que é necessário o reconhecimento precoce do risco cardiovascular.

Diante da abordagem do manuseio correto das ações proporcionadas ao cuidado pode se constatar que a enfermagem tem um papel importante na avaliação e controle dos efeitos colaterais sofridos pelo paciente submetido à quimioterapia.

É de fundamental relevância que a enfermagem atue antes, durante e após a administração da quimioterapia, observando os sinais e sintomas e orientando ambos tanto o paciente quanto o familiar sobre o antineoplásico utilizado, sua ação no organismo, seus efeitos colaterais e os cuidados em geral.

Baseado nos achados, o artigo contribuiu para o conhecimento dos quimioterápicos cardiotóxicos, tornando-se uma ferramenta fundamental para elaboração de um plano assistencial, contribuindo para uma assistência à saúde eficaz e de qualidade ao paciente.

\section{Referências}

Adão, R., Keulenaerb G., Moreira A. L., \& Silva C. B. 2013. Cardiotoxicidade associado à terapêutica oncológica: mecanismos fisiopatológicos e estratégias de prevenção. Revista Portuguesa de Cardiologia, 32(5): 395-409.

Bonassa, E. M. A, \& Gato M. I. R. 2012. Terapêutica oncológica para enfermeiros e farmacêuticos. São Paulo, SP: Atheneu.

Borges, M. D., Silva, R. D. O., Andrade, K. B. S., Pereira, S. R. M., Almeida, L. F., \& Mendonça, H. D. 2018. Avaliação dos registros de enfermagem acerca das manifestações clínicas de cardiotoxicidade. Revista Enfermagem Atual, 86(24):1-10.

Brasil, Ministério da Saúde. 2006. A situação do câncer no Brasil. Rio de Janeiro, RJ: INCA.

Cruz, M., Rodrigues, J. D., \& Campelo, M. 2016. Cardiotoxicidade na terapêutica com antraciclinas: estratégias de prevenção. Revista Portuguesa de Cardiologia, 35(6):359-71.

Fassio, R. C. C., \& Castillo, J. M. D. 2017. Aplicação da ecocardiografia na detecção precoce de cardiotoxicidade por quimioterapia. Arquivo Brasileiro de Cardiologia: Imagem cardiovasc, 30(1): 8-12.

Filho, A. C. A., \& Gonçalves, M. J. F. 2011. Cardiologia e oncologia: uma visão interdisciplinar. Saúde \& Transformação Social, 1(2), 150-154.

Filho, R. K., Hajjar, L. A., Bacal, F., Hoff, P. M., Diz, M. D. P., \& Galas, F. R. B. G. 2011. I Diretriz Brasileira de cardio-oncologia da Sociedade Brasileira de Cardiologia. Arquivos Brasileiros de Cardiologia, 96(2), 01-52.

Gil, A. C. 1994. Como elaborar projetos de pesquisa (3a ed.). São Paulo, SP: Atlas.

Gil, A. C. 2002. Como elaborar projetos de pesquisa (4a ed.). São Paulo, SP: Ed. Atlas.

Gomes, I. S., \& Caminha, I. O. 2014. Guia para estudos de revisão sistemática: uma opção metodológica para as ciências do movimento humano. Movimento, Porto Alegre,20(1), 395-411.

Instituto Nacional de Câncer José Alencar Gomes da Silva. 2020. ABC do câncer: abordagens básicas para o controle do câncer. Rio de Janeiro, RJ: INCA.

Jesus, E. B. 2019. Protocolo assistencial de enfermagem para avaliação e manejo de pacientes adultos submetidos a terapia oncológica cardiotóxica: estudo metodológico. Niterói,RJ.

Morais, G. B., Oliveira, E. P., Alexandre, J. K. L., Ferreira, L. H. R. M., Cruz, M. R., \& Cavalcanti, T. R. F. 2018. A valia do vínculo na relação equipe multidisciplinar-paciente oncológico para a continuidade do cuidado: uma revisão integrativa. Revista Saúde \& Ciência online, 7(2), 502.

Zata, S. M., Alves, B. C. A., Bacci, M. R., Murad N., Feder D., Gehrke, F. S., ... Fonseca, F. L. A. 2014. Uso do pré-condicionamento isquêmico na oncologia pelo profissional de enfermagem. Rev. Enfermagem Revista, 17(2), 128-136. 


\section{Minicurrículo}

Lorena Esmeralda Nascimento Celeste.Possui graduação em Enfermagem pelo Centro Universitário Jorge Amado (UNIJORGE, 2012). Especialista em Atenção Básica pela Universidade Federal do Maranhão (UFMA, 2015). Especialista em Docência do Ensino Superior (UNIFACS, 2020). Enfermeira do Programa de Valorização do Profissional da Atenção Básica (PROVAB, 2014). Enfermeira Preceptora na Universidade Salvador.

Maiara Rodrigues Maia.Possui graduação em Enfermagem pelo Centro Universitário Jorge Amado (2012). Especialista em Enfermagem em Emergência pelo Centro Universitário Estácio/FIB (2015). Especialista em EPS em Movimento pelo UFRGS (2015). Especialista em micropolítica da gestão e trabalho em saúde pela UFF (2018). Especialista em Enfermagem oncológica (2019). Enfermeira do Programa de saúde da família do município de Itaetê-Ba (2014), Enfermeira do Programa de saúde da família do município de Utinga-Ba (2015). Experiência em atendimento ao paciente em doenças infectocontagiosas. Residente no programa de residência multiprofissional do hospital de beneficências de senhoras Sírio Libanês no programa de cuidado do paciente oncológico.

\footnotetext{
Como citar: Celeste, L.E.N., \& Maia, M.R. 2021. Cuidados de enfermagem relacionados à cardiotoxicidade envolvendo drogas como a antraciclinas e anticorpo monoclonais no tratamento oncológico.Pubsaúde, $\quad 5, \quad$ a095. DOI: https://dx.doi.org/10.31533/pubsaude5.a095

Recebido:3 nov. 2020.

Revisado e aceito: 16 nov. 2020

Conflito de interesse: os autores declaram, em relação aos produtos e companhias descritos nesse artigo, não ter interesses associativos, comerciais, de propriedade ou financeiros que representem conflito de interesse.

Licenciamento: Este artigo é publicado na modalidade Acesso Aberto sob a licença CreativeCommons Atribuição 4.0 (CC-BY 4.0).
} 